\title{
Contribution of geometry of interaction between interplanetary and terrestrial magnetic fields into geomagnetic activity
}

\author{
T. V. Kuznetsova, A. I. Laptukhov, and V. D. Kuznetsov \\ IZMIRAN, Russian Academy of Sciences, Moscow region, Troitsk, 142190, Russia \\ email: tvkuz@izmiran.ru
}

\begin{abstract}
We present results of our study of dependence of planetary geomagnetic activity from geometric factors in geoeffective parameters taking into account orientation of the geomagnetic moment $\mathrm{M}$ relative to the vectors of the Interplanetary Magnetic Field (IMF) and electric field of the solar wind E during annual and daily motions of the Earth. We take as our data base space measurements of the IMF and solar wind velocity at the Earth's orbit for 1964-1998 and Kp, Dst indices. Variations of the geometric factors determined by mutual orientation of the vectors $\mathrm{E}$ and $\mathrm{M}$ can explain $50 \%$ of observed variations of $\mathrm{Kp}$ and $75 \%$ of Dst. We show that geomagnetic activity can reach very high levels of geomagnetic activity $\mathrm{Kp}=8$ for invariable values of the solar wind electric field by changing only geometric factors.
\end{abstract}

Keywords. Sun: solar-terrestrial relations

Geomagnetic activity is determined in our approach by two kinds of causes: time variations of the solar wind parameters and IMF, sources of which are time variations of solar activity and by time variations of mutual orientation of geomagnetic moment $\mathbf{M}$, IMF $\mathbf{B}$, electric field of the solar wind $\mathbf{E}$, causes of which are mainly annual and daily motions of the Earth. We considered the geometry in scope of a reconnection model taking into account effects of orbital and daily motions of the magnetic moment of the Earth (Kuznetsova et al. 2002). Based on the model we suggested geoeffective independent invariant (relative to a coordinate system) parameters. We showed that changes of these parameters can explain $95 \%$ of observed variations of $\mathrm{Kp}$ index of planetary geomagnetic activity (Kuznetsova et al. 2006). We did not divide in this study influence of changes of values and geometric factors on $\mathrm{Kp}$ variations that is aim of the present study.

We assume that reconnection in general case of arbitrary oriented fields is determined by the following parameters (Kuznetsova et al. 2002):

$$
\mathrm{Bm}=(\mathbf{B}, \mathbf{M})=\mathrm{BxMx}+\mathrm{ByMy}+\mathrm{BzMz}=|\mathbf{B}| \cdot|\mathbf{M}| \cdot \cos (\alpha),
$$

where $\mathrm{Bm}$ is a component of the IMF vector $\mathbf{B}$ directed along the dipole magnetic moment vector $\mathbf{M}, \alpha$ is an angle between the vectors of $\mathbf{M}$ and $\mathbf{B},|\mathbf{B}|$ and $|\mathbf{M}|$ are values of the vectors; $\mathrm{Bi}, \mathbf{M i}$ are components of vectors $\mathbf{B}$ and $\mathbf{M}$ in a coordinate system.

$$
\mathrm{Em}=(\mathbf{E}, \mathbf{M})=\mathrm{V}(\mathrm{MzBy}-\mathrm{MyBz})=|\mathbf{E}| \cdot\left[(\mathrm{My})^{2}+(\mathrm{Mz})^{2}\right]^{1 / 2} \cdot \cos (\text { Uem }),
$$

where $\mathrm{Em}$ is a component of the solar wind electric field $\mathbf{E}=[\mathbf{B}, \mathbf{V}]$ along vector $\mathbf{M}$, $\mathbf{V}$ is vector of velocity oriented along axis $-\mathrm{X}$ (here and below we use GSE coordinate system), $\mathrm{V}=|\mathbf{V}|$ is its value, Uem is angle between vector $\mathbf{E}$ and projection of $\mathbf{M}$ on plane YZ. To calculate geometric factors of interaction from (1), (2) it is necessary to know components of the vectors $\mathbf{B}, \mathbf{V}$ and $\mathbf{M}$. We calculated orientation of $\mathbf{M}$ in GSE c.s. for any UT of a year. We use mean hourly measurements of the solar wind velocity $\mathrm{V}$ and IMF in GSE c.s. at the Earth's orbit for the period 1964-1998 and data of planetary geomagnetic index Kp (http://www.nssdc.nasa.gov/omniweb). Kp is given here in a form 

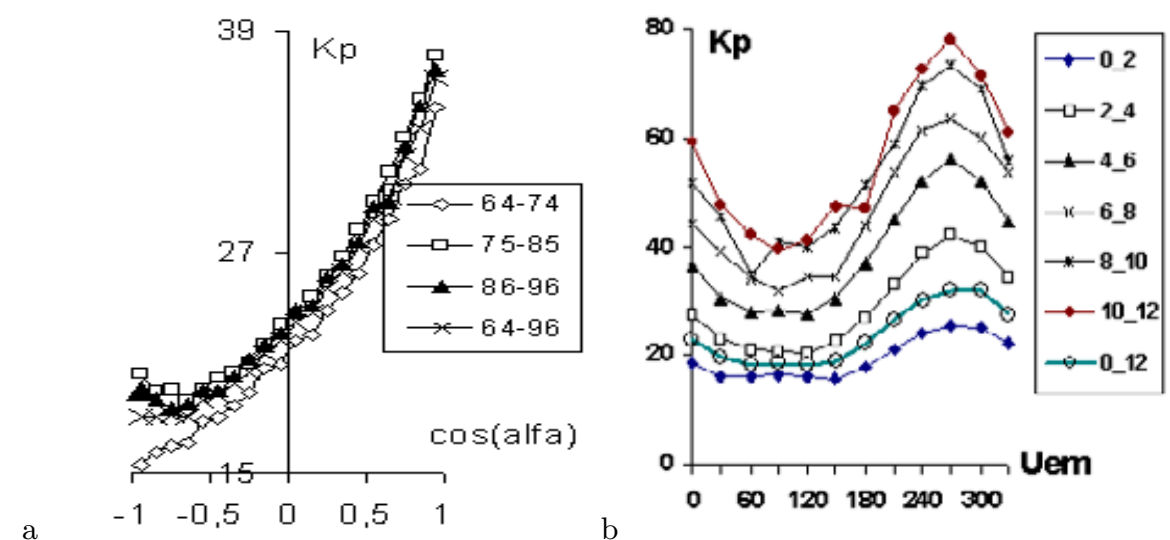

Figure 1. Influence of geometric parameters on $\mathrm{Kp}$ : (a) dependence $\mathrm{Kp}$ from $\cos (\alpha)$ for different intervals of years indicated in column, Cc is correlation coefficient between the data from different intervals of years; (b) dependence $\mathrm{Kp}$ from angle Uem (degrees) for different ranges of $\mathrm{E}$ values $(\mathrm{mv} / \mathrm{m})$ shown in column.

comfortable for calculations that we use in this paper: $(3+=33,6-=57,4=40)$, instead of usual changes of Kp in range 0-9 with step 1/3. Results of our studies of influence of geometric parameters on Kp are shown on figure 1 . We see at figure 1(a) that during turn of the IMF from northward direction along vector $\mathbf{M}(\cos (\alpha)=-1)$ to southward one $(\cos (\alpha)=1) \mathrm{Kp}$ changes from 15 to $40, \Delta \mathrm{Kp}=25$ that is equaled to $28 \%$ from variations $\mathrm{Kp}$. Curves $\mathrm{Kp}$ from $\cos (\alpha)$ for different years are correlated with high coefficient Cc. Thus, $\cos (\alpha)$ is geometric factor repeating from year to year and influencing $\mathrm{Kp}$. We see at figure $1(\mathrm{~b})$ that for Uem $=0^{\circ}(\mathbf{E}$ is oriented along $\mathbf{M})$ and for Uem $=180^{\circ}$ (directions of $\mathbf{E}$ and $\mathbf{M}$ are opposite) Kp has the mean values within each fixed range of $\mathbf{E}$. This conclusion is in agreement with our previous result that sign of component Em does not influence on Kp changes (Kuznetsova et al. 2002) that allowed us to connect the Em field with stationary magnetospheric convection independenting from sign of By component of IMF. For Uem $=90^{\circ}$ ( $\mathbf{E}$ is perpendicular to $\mathbf{M}$, direction to dawn) Kp has minimum, for $\mathrm{Uem}=270^{\circ}$ ( $\mathbf{E}$ is perpendicular to $\mathbf{M}$, direction to dusk) Kp has maximum. The geometric effect is larger for larger values of $\mathrm{E}$. A remarkable feature of sine curve at figure 1 (b) for range $\mathrm{E}=10-12 \mathrm{mv} / \mathrm{m}$ is that $\mathrm{Kp}$ changes from $\mathrm{Kp}=40$ (minimum) to $\mathrm{Kp}=80$ (maximum), $\Delta \mathrm{Kp}=40$ (50\% of observed statistical variations of $\mathrm{Kp}$ ). Similar geometric effect in Dst index is equalled to $75 \%$ (it is not shown from limited volume of the paper). Thus, time changes of the $\mathrm{E}$ values are responsible for the rest $\sim 50 \%$ and $25 \%$ of the observed variations of $\mathrm{Kp}$ and Dst accordingly. This means that geomagnetic activity can reach very high level $\mathrm{Kp}=8$ (Dst $=-180 \mathrm{nT}$ ) for invariable values of $\mathrm{E}$ in the solar wind from geomagnetic active state $\mathrm{Kp}=4$ (Dst $=-30 \mathrm{nT}$ ) by changing only angle between $\mathrm{E}$ and $\mathrm{M}$ (Kp in usual units). We used for receiving the figure 1 (b) about 150,000 hourly values of IMF and V that points to statistical realiability of our results.

The work is supported by Russian Fund of Basic Researches via grant 06-05-64998.

\section{References}

Kuznetsova, T.V. \& Laptukhov, A.I. 2004, in: W. Shroeder (ed.), New and Old Problems in Physics and Geophysics (Bremen: Science), p. 10

Kuznetsova, T.V., Laptukhov, A.I., \& Kuznetsov, V.D. 2006, Astron. Vestnik, 4, 17 (in Russian) 